\title{
Distribution-Free Learning of Bayesian Network Structure
}

\author{
Xiaohai Sun \\ Max Planck Institute for Biological Cybernetics \\ Spemannstr. 38, 72076 Tübingen, Germany \\ xiaohai.sun@tuebingen.mpg.de
}

\begin{abstract}
We present an independence-based method for learning Bayesian network (BN) structure without making any assumptions on the probability distribution of the domain. This is mainly useful for continuous domains. Even mixed continuous-categorical domains and structures containing vectorial variables can be handled. We address the problem by developing a non-parametric conditional independence test based on the so-called kernel dependence measure, which can be readily used by any existing independence-based BN structure learning algorithm. We demonstrate the structure learning of graphical models in continuous and mixed domains from real-world data without distributional assumptions. We also experimentally show that our test is a good alternative, in particular in case of small sample sizes, compared to existing tests, which can only be used in purely categorical or continuous domains.
\end{abstract}

Keywords: graphical models, independence tests, kernel methods.

\section{Introduction}

There are two basic classes of BN learning algorithms, namely constraint-based (or independence-based) and model-based approaches. The model-based approaches often employ a search in the space of all possible structures guided by a heuristic function, usually penalized log-likelihood or Bayesian metric [12]3. They are also called score-based approach in many literature. One of the challenges of applying score-based methods is the assessment of informative priors on possible structures and on parameters for those structures. Constraint-based approaches carry out conditional independence (CI) tests on the database and build a BN structure in agreement with the obtained independence restrictions. They make weak commitments as to the nature of relationships between variables, i.e., faithfulness/stability 4 45]. The best-known example of this kind of approaches is the so-called PC algorithm 4. The output of PC is the Markov equivalence class of BN structures, which can often be interpreted as causal graph under some additional technical assumptions, e.g., causal sufficiency. Such independence-based algorithms can essentially be broken into an adjacency phase and an orientation phase. The rule of learning adjacency is to test whether a set variables $Z$ 

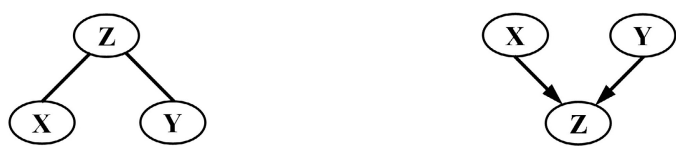

Fig. 1. The " $\wedge$ "-structure (left) represents the Markov equivalence class of " $X \leftarrow Y \rightarrow$ $Z$ ", " $X \rightarrow Y \rightarrow Z$ ", and " $X \leftarrow Y \leftarrow Z$ ", where constraints $X \not \Perp Y$ and $X \Perp Y \mid Z$ hold by construction. In the " $\mathrm{V}$ "-structure (right), $X \Perp Y$ and $X \not \Perp Y \mid Z$ hold by construction

exists which makes marginally dependent $X$ and $Y$ conditionally independent, denoted by $X \not \Perp Y$ and $X \Perp Y \mid Z$ (the so-called " $\wedge$ "-structure in Fig. 1] left). The orientation strategy bases on the so-called collider (" $\vee$ "structure) identification, namely if some subset $S$ exists such that $X \Perp Y \mid S$ and $X \not \Perp Y \mid(S, Z)$, a $\vee$-structure (Fig. 1, right) can be inferred. Consequently, the accuracy and reliability of CI tests play a central role in independence-based algorithms.

One of the crucial problems of learning BN structure from data is making a choice on the kind of probability distributions. Commonly, a local probability distribution function (PDF) needs to be defined between every variable and its parents. Such distributions are used to model local interactions among subsets of variables in the model. CI tests that do not assume any particular family of distributions are called non-parametric. Although such tests exist for categorical variables, e.g., $\chi^{2}$ test or test via mutual information (MI) is perhaps the most common one, the problem in continuous or mixed continuous-categorical domains is considerably harder. The common assumption is for the local PDFs between parents and children to be linear relations with additive Gaussian errors, as the correlation analysis in PC algorithm. However, there are many situations where this assumption fails and the underlying interactions are far from linear. In some situation, interactions cannot be captured by correlation analysis at all. To make this apparent, we demonstrate the following toy data example. We sampled dataset $\left(X_{0}, Y_{0}\right)$ as shown in the leftmost plot of Fig. 2 and then transformed the original dataset by a rotation of angle $\omega$ (in degree) into a new dataset $\left(X_{\omega}, Y_{\omega}\right)$. The second and the third plot (from left) in Fig. 2 visualize the transformed data $\left(X_{45}, Y_{45}\right)$ and $\left(X_{90}, Y_{90}\right)$ respectively. According to the generating model with $P\left(X_{0} \mid Y_{0}<0\right)=P\left(X_{0} \mid Y_{0} \geq 0\right)$ (see Fig. 2 for the description of the underlying model), $X_{0}$ and $Y_{0}$ are independent. It is obvious that $X_{\omega}$ and $Y_{\omega}$ are only independent for $\omega=0,90,180, \ldots$.

It is easy to see that the correlation matrix $\rho_{0}$ of the data matrix

$$
\mathcal{D}_{0}:=\left(\begin{array}{c}
X_{0} \\
Y_{0}
\end{array}\right) \text { is a unit matrix, namely } \rho_{0}:=\left(\begin{array}{cc}
\rho_{X_{0} X_{0}} & \rho_{X_{0} Y_{0}} \\
\rho_{Y_{0} X_{0}} & \rho_{Y_{0} Y_{0}}
\end{array}\right)=\left(\begin{array}{ll}
1 & 0 \\
0 & 1
\end{array}\right) .
$$

Further, it is well known that the rotation matrix $R_{\omega}$ of angle $\omega$ in an anticlockwise direction has the form of

$$
R_{\omega}=\left(\begin{array}{r}
\cos \left(\frac{\pi}{180} \omega\right) \sin \left(\frac{\pi}{180} \omega\right) \\
-\sin \left(\frac{\pi}{180} \omega\right) \cos \left(\frac{\pi}{180} \omega\right)
\end{array}\right) \text {. Hence, }\left(\begin{array}{c}
X_{\omega} \\
Y_{\omega}
\end{array}\right)=: \mathcal{D}_{\omega}=R_{\omega} \mathcal{D}_{0}=R_{\omega}\left(\begin{array}{c}
X_{0} \\
Y_{0}
\end{array}\right)
$$



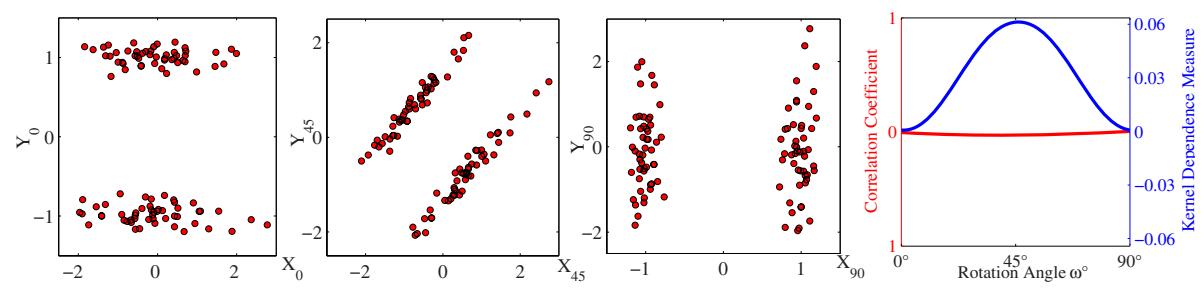

Fig. 2. 100 data points of $Y_{0}$ is sampled from $\frac{1}{2} \mathcal{N}(1,0.01)+\frac{1}{2} \mathcal{N}(-1,0.01)$, where $\mathcal{N}\left(\mu, \sigma^{2}\right)$ denotes a Gaussian distribution with expectation $\mu$ and variance $\sigma^{2}$. Data points of $X_{0}$ are sampled from $P\left(X_{0} \mid Y_{0}<0\right) \propto \mathcal{N}(0,1)$ and $P\left(X_{0} \mid Y_{0} \geq 0\right) \propto \mathcal{N}(0,1)$. $\left(X_{\omega}, Y_{\omega}\right)$ is transformed dataset with rotation angle $\omega$ in an anticlockwise direction. $X_{0}$ and $Y_{0}$ (leftmost plot) as well as $X_{90}$ and $Y_{90}$ (second plot from right) are mutually independent by construction, whereas $X_{45}$ and $Y_{45}$ (second plot from left) are strongly dependent due to the rotation angle 45 . The rightmost plot visualizes the typical curve of the empirical correlation coefficients (red line) for $\omega \in[0,90]$, and a typical curve of the empirical kernel dependence measures (blue line), which will be defined in the next section. Correlation coefficient vanishes for any $\omega$. Kernel dependence measures seem to capture the magnitude of dependences reasonably.

depicts the dataset transformed by a rotation angle $\omega$. The corresponding correlation matrix $\rho_{\omega}$ is given by

$$
\rho_{\omega}=\mathrm{E}\left[R_{\omega}\left(\mathcal{D}_{0}-\mathrm{E}\left[\mathcal{D}_{0}\right]\right)\left(\mathcal{D}_{0}-\mathrm{E}\left[\mathcal{D}_{0}\right]\right)^{\mathrm{T}} R_{\omega}^{\mathrm{T}}\right]=R_{\omega} \rho_{0} R_{\omega}^{\mathrm{T}}=\rho_{0}
$$

where "E[.]" depicts the expectation. This means that the correlation coefficient vanishes for an arbitrary $\omega$, while the dependence indeed vanishes only for few specific rotation angles $\omega=0,90,180, \ldots$. This is the reason why it is not surprising that the performance of the conventional BN learning algorithms, e.g. PC, is sometimes unsatisfactory, since it takes only linear interactions into account.

A non-parametric Bayesian method of testing independence on continuous domains is recently proposed by Margaritis et al. 677. Their method is not based on a conventional hypothesis test but on the calculation of probability of independence given data by the Bayesian approach. To determine whether two variables are (conditionally) independent, they discretized the domains by maximizing the posterior probability of dependence given data. If the probability of independence $P$ (Independence) larger than $\frac{1}{2}$, the independence is verified, otherwise dependence. More precisely, the method determines the probability of dependence by calculating the likelihoods of modeling the data as dependent with a joint multinomial distribution or as independent with two marginal multinomial distribution. Margaritis' Bayesian method is impressive because it is the first practicable distribution-free learning of BN structure in purely continuous domains, although it involves a sophisticated process of domain discretization. We will compare our test method with this method later. 


\section{Kernel Tests of Independence}

Since kernel methods [9] can transform the non-linear relationship into a linear one in the feature space, we use the kernel-based dependence measure introduced by [10] as the general framework for our tests. First, we recall some standard definitions. A positive definite kernel $k_{\mathcal{X}}: \mathcal{X} \times \mathcal{X} \rightarrow \mathbb{R}$ on some non-empty set $\mathcal{X}$ is a symmetric function, i.e., $k_{\mathcal{X}}\left(x, x^{\prime}\right)=k_{\mathcal{X}}\left(x^{\prime}, x\right)$ for any $x, x^{\prime} \in \mathcal{X}$ such that for arbitrary $n \in \mathbb{N}$ and $x^{(1)}, \ldots, x^{(n)} \in \mathcal{X}$ the matrix $K$ with $(K)_{i j}:=k_{\mathcal{X}}\left(x^{(i)}, x^{(j)}\right)$ is positive definite, i.e., $\sum_{i, j=1}^{n} c_{i} c_{j} k_{\mathcal{X}}\left(x^{(i)}, x^{(j)}\right) \geq 0$ for all $c_{1}, \ldots, c_{n} \in \mathbb{R}$. A reproducing kernel Hilbert space $(\mathrm{RKHS}) \mathcal{H}_{\mathcal{X}}$ is a Hilbert space defined by the completion of an inner product space of functions $k_{\mathcal{X}}(x, \cdot)$ with $x \in \mathcal{X}$ and the inner product defined by $\left\langle k_{\mathcal{X}}(x, \cdot), k_{\mathcal{X}}\left(x^{\prime}, \cdot\right)\right\rangle=k_{\mathcal{X}}\left(x, x^{\prime}\right)$ for all $x, x^{\prime} \in \mathcal{X}$. In other words, $\Phi(x)(\cdot)=k_{\mathcal{X}}(x, \cdot)$ defines a map from $\mathcal{X}$ into a feature space $\mathcal{H}_{\mathcal{X}}$. With the so-called "kernel trick", a linear algorithm can easily be transformed into a non-linear algorithm, which is equivalent to the linear algorithm operating in the space of $\Phi$. However, because the kernel function is used for calculating the inner product, the mapping $\Phi$ is never explicitly computed. This is desirable, because the high-dimensional space may be infinite-dimensional, as is the case when the kernel, e.g., is a Gaussian: $k_{\mathcal{X}}: \mathbb{R}^{m} \times \mathbb{R}^{m} \rightarrow \mathbb{R}, k_{\mathcal{X}}\left(x, x^{\prime}\right)=$ $\exp \left(-\left\|x-x^{\prime}\right\|^{2} / 2 \sigma^{2}\right)$.

Within the kernel framework, the conditional cross-covariance operator expressing correlations between variables in the RKHS can be introduced. Let $\left(\mathcal{H}_{\mathcal{X}}, k_{\mathcal{X}}\right),\left(\mathcal{H}_{\mathcal{Y}}, k_{\mathcal{Y}}\right),\left(\mathcal{H}_{\mathcal{Z}}, k_{\mathcal{Z}}\right)$ be RKHSs on measurable spaces $\mathcal{X}, \mathcal{Y}, \mathcal{Z}$ respectively, $(X, Y, Z)$ be a random vector on $\mathcal{X} \times \mathcal{Y} \times \mathcal{Z}$. For all $f \in \mathcal{H}_{\mathcal{X}}$ and $g \in \mathcal{H}_{\mathcal{Y}}$, there exists (under some technical assumptions [1]) a unique conditional crosscovariance operator $\Sigma_{Y X \mid Z}$ such that $\left\langle g, \Sigma_{Y X \mid Z} f\right\rangle_{\mathcal{H}_{\mathcal{Y}}}=\mathrm{E}_{Z}[\operatorname{Cov}[f(X), g(Y) \mid Z]]$. If the RKHSs are induced by characteristic kernels [11, e.g., Gaussian kernels, vanishing of the operator is equivalent to the conditional independence [10]: $\Sigma_{\ddot{Y} \ddot{X} \mid Z}=O \Leftrightarrow X \Perp Y \mid Z$ where $\ddot{X}:=(X, Z)$ and $\ddot{Y}:=(Y, Z)$. It should be stressed that $X, Y, Z$ can be continuous, categorical, vectorial or mixed. For strictly nominal-categorical variables, the natural way to represent the $d$ nominal alternatives, namely $d$ unit vectors in a $d$-dimensional Cartesian coordinate system: $\left\{(1,0, \ldots)^{\mathrm{T}},(0,1, \ldots)^{\mathrm{T}}, \ldots,(\ldots, 0,1)^{\mathrm{T}}\right\} \subset \mathbb{R}^{d}$.

Following the work of [1210], we evaluate the operator with Hilbert-Schmidt (HS) norm and denote the empirical estimator by $\widehat{\mathbb{H}}_{Y X \mid Z}:=\left\|\Sigma_{\ddot{Y} \ddot{X} \mid Z}\right\|_{\mathrm{HS}}^{2}$. All these statements hold also for the unconditional operator and marginal independence $\Sigma_{Y X}=O \Leftrightarrow X \Perp Y$. Given RKHS induced by Gaussian kernels, where functions being less smooth correspond to larger RKHS-norms, large values will then indicate correlations between smooth functions of $X$ and $Y$. A finite cut-off value for the kernel dependence measure corresponds to neglecting correlations if they are small or if they occur only on complex (not sufficiently smooth) functions. Instead of thresholding the kernel measure directly as in our previous work [10, we employ permutation tests [13] for judging whether dependencies are significant or not. Although permutation tests require a large number of calculations of HS-norms, the computation is feasible if we use the incomplete Cholesky 
decomposition $\widehat{K}=L L^{\mathrm{T}} 14$ where $L$ is a lower triangular matrix determined uniquely by this equation. This may lead to considerably fewer columns than the original matrix. If $k$ columns are returned, the storage requirements are $O(k n)$ instead of $O\left(n^{2}\right)$, and the running time of many matrix operations reduces to $O\left(n k^{2}\right)$ instead of $O\left(n^{3}\right)$. An alternative kernel statistical test based on moment matching is currently proposed by Gretton et al. [15. Instead of computing the HS-norm of the operator directly, they designed a test statistics based on entries of kernel matrices. But, for one thing, this alternative test is designed for unconditional cases, because the null distribution only in such cases is known. For the other thing, they claimed that the permutation test may outperform their alternative in some situation, particularly if the sample size is small (e.g., less than 200), since the estimation of second moments of entries of kernel matrices tends to be unreliable (see [15] for experiments with text data).

Given a data matrix $(X, Y, Z)$ with $X:=\left(x^{(1)}, \ldots, x^{(n)}\right)^{\mathrm{T}}$ and so on, testing unconditional independence $X \Perp Y$ via random permutations is straightforward. But how to approximate the null distribution of the kernel dependence measure $\widehat{\mathbb{H}}_{Y X \mid Z}$ under conditional independency is non-trivial. The idea is that random permutations should release the connection between $X$ and $Y$ to simulate the independency. On the other hand, the mutual relations between $X$ and $Z$ and between $Y$ and $Z$ have to be kept, since $Z$ is tied to a specific value. In order to yield this effect, we propose to restrict the random permutations $\pi$ to those that satisfy the condition $z^{(\pi(i))}=z^{(i)}$ for $i=1, \ldots, n$. In other words, if $Z$ is categorical, $\pi$ is restricted to random permutations within the same category of $Z$. If $Z$ is real-valued or vectorial, the condition could be said to hold if $z^{\left(\pi_{j}(i)\right)}$ and $z^{(i)}$ are "similar" in some sense. This suggests the use of clustering techniques to search for an appropriate partition of $Z$. Then, the data points within the same partition are similar w.r.t. values of $Z$ (see Tab. 1). This way, we designed a kernel independence test providing a general non-parametric tool for verifying CI constraints in both conditional and unconditional cases. In our following experiments, we employed the standard k-means clustering to construct the partition of data points of conditioning variables and computed the approximate null distribution under conditional independency. The significance level is set to be 0.05 throughout this paper.

Table 1. Set of random permutations that are used for the test of conditional independence $X \Perp Y \mid Z$. $Z$ data are clustered into $n$ partition. Within each partition $z^{(i)}$, generate simulated conditionally independent data $\left(X^{\pi_{j}}, Y\right)$.

\begin{tabular}{|c|c|c|c|c|c|c|}
\hline & partition $z^{(1)}$ & partition $z^{(2)}$ & $\ldots$ & $\ldots$ & $\ldots$ & partition $z^{(n)}$ \\
\hline$Z$ & $z^{\left(1_{1}\right)} z^{\left(1_{2}\right)} \cdots z^{\left(1_{m}\right)}$ & $z^{\left(2_{1}\right)} z^{\left(2_{2}\right)} \cdots z^{(2 m)}$ & $\ldots$ & $\ldots$ & $\cdots$ & $z^{\left(n_{1}\right)} z^{\left(n_{2}\right)} \cdots z^{\left(n_{m}\right)}$ \\
\hline$Y$ & $y^{\left(1_{1}\right)} y^{\left(1_{2}\right)} \cdots y^{\left(1_{m}\right)}$ & $y^{\left(2_{1}\right)} y^{\left(2_{2}\right)} \cdots y^{(2 m)}$ & $\ldots$ & $\cdots$ & $\cdots$ & $y^{\left(n_{1}\right)} y^{\left(n_{2}\right)} \cdots y^{\left(n_{m}\right)}$ \\
\hline$X^{\pi_{j}}$ & $x^{\left(1_{1}\right)} x^{\left(1_{2}\right)} \cdots x^{\left(1_{m}\right)}$ & $x^{\left(2_{1}\right)} x^{\left(2_{2}\right)} \cdots x^{(2 m)}$ & $\ldots$ & $\ldots$ & $\cdots$ & $x^{\left(n_{1}\right)} x^{\left(n_{2}\right)} \cdots x^{\left(n_{m}\right)}$ \\
\hline
\end{tabular}




\section{Experiments}

Learning BN structure in real applications is challenging, because real-world data are often on mixed domains and the relationships are often nonlinear. Sometimes, the real data even do not necessarily have the representation of a BN structure. The conventional approaches either can not handle them or ignore possible problems. By means of some benchmark real-world data, we will show some respects, in which some improvements can be achieved by our method in comparison to conventional ones, in particular in relation to causal structure learning, to which the independence-based approaches are supposed to be often applied.

\subsection{Real-World BN Structure Learning}

Digoxin Clearance. The study of the passage of drugs through the body is of essential interest in medical science. A real-world dataset on 35 consecutive patients under treatment for heart failure with the drug digoxin is analyzed in [16] (see also 17 p. 323 and [18 p. 42). The renal clearances of digoxin, creatinine, and urine flow were determined simultaneously in each of the patients receiving digoxin, in most of whom there was prerenal azotemia. Halkin et al. [16] and Edwards [18] based their analysis on the (partial) correlation coefficient. In comparison to the correlation analysis and Margaritis' Bayesian method, we conducted the kernel tests (see Tab. 2 for results). A visual inspection of the data indicates that the linearity assumption appears to be reasonable for the dependence between the creatinine and digoxin clearances (Fig. 3, leftmost). A linear relation between them was first suggested by Jelliffe et al. [19] and later confirmed by various clearance studies, which revealed a close relationship between creatinine and digoxin clearance in many patients. The ready explanation is that both creatinine and digoxin are mainly eliminated by the kidneys. In agreement with this explanation, all three tests found the unconditional and conditional dependence (Tab. 2, first and second row).

As one can see from Fig. 3, the relations between creatinine clearance and urine flow (second plot) and between digoxin clearances and urine flow (third plot) are less linear than the relation between creatinine and digoxin clearance
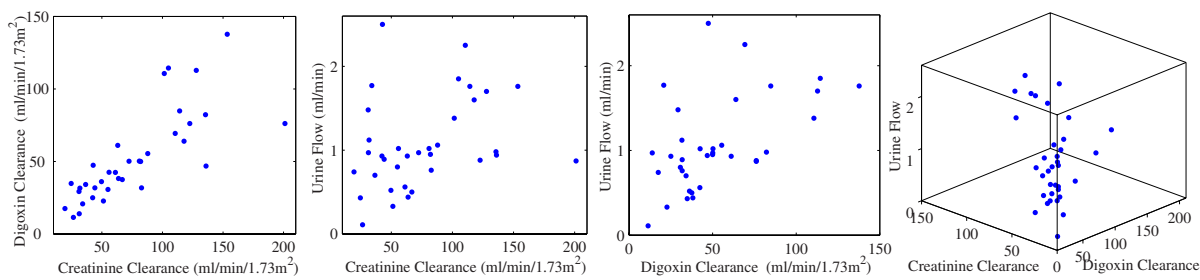

Fig. 3. Data on 35 consecutive patients under treatment for heart failure with the drug digoxin. Clearances are given in $\mathrm{ml} / \mathrm{min} / 1.73 \mathrm{~m}^{2}$, urine flow in $\mathrm{ml} / \mathrm{min}$. 
Table 2. Comparison of independence tests on digoxin clearance data. Significance level $\alpha=0.05$ is chosen for analysis via correlations and kernel dependence measures.

\begin{tabular}{|l||c|c||c|c||c|c|}
\hline \multirow{2}{*}{\multicolumn{1}{|c||}{ Independence Hypothesis }} & \multicolumn{2}{c||}{ Correlation } & \multicolumn{2}{c||}{ Margaritis' Bayesian } & \multicolumn{3}{c|}{ Kernel Dependence } \\
\cline { 2 - 7 } & p-value & Test & $P$ (Independence) & Test & p-value & Test \\
\hline Creatinine $\Perp$ Digoxin & 0.00 & Reject & 0.0030 & Reject & 0.00 & Reject \\
\hline Creatinine $\Perp$ Digoxin $\mid$ Urine Flow & 0.00 & Reject & 0.1401 & Reject & 0.00 & Reject \\
\hline Creatinine $\Perp$ Urine Flow & 0.07 & Accept & 0.7018 & Accept & 0.01 & Reject \\
\hline Creatinine $\Perp$ Urine Flow $\mid$ Digoxin & 0.40 & Accept & 0.8538 & Accept & 0.58 & Accept \\
\hline Digoxin $\Perp$ Urine Flow & 0.00 & Reject & 0.0474 & Reject & 0.00 & Reject \\
\hline Digoxin $\Perp$ Urine Flow $\mid$ Creatinine & 0.02 & Reject & 0.7851 & Accept & 0.17 & Accept \\
\hline
\end{tabular}

(first plot). The correlation analysis (see also 18 p. 43) did not reveal the dependence between creatinine clearance and urine flow, whereas kernel test did (third row of Tab. 22). We conjecture that Margaritis' Bayesian method failed to detect dependence in this case because of the small sample size, which gives evidence for better performance of kernel tests in case of small datasets. All three tests found that, given digoxin clearance, creatinine clearance was not significantly related to urine flow rate (Tab. 2] fourth row). All methods found that in these patients digoxin clearance was significantly related to urine flow rate (Tab. 2, fifth row). This finding is consistent with the opinion of Halkin et al. 16, who suspected that the elimination of digoxin might be subject to reabsorption, which might give rise to a correlation with urine flow. However, if the linear dependence model is wrong, a biased estimate of the partial correlation and a biased test for independence via linear model may result. Both non-parametric tests, i.e., kernel tests and Margaritis' Bayesian method accepted the hypothesis that, given creatinine clearance, digoxin clearance is independent of urine flow, whereas the partial correlation did not confirm this hypothesis (last row of Tab. 2). The finding that digoxin clearance is independent of urine flow controlling for creatinine clearance is particularly of medical interest. In summary, the test results revealed that the non-parametric tests are superior to correlation analysis. This example makes also clear that, in practice, independence by kernel measures does not necessarily require the independence by correlation analysis, although it is theoretically apparent that non-vanishing of correlation implies non-vanishing of kernel dependence measure.

Even though all CI constraints are correctly detected by some test, we can have problem to learn BN structures. The problem is that the CI constraints could be inconsistent w.r.t. representation by BN structures. In this example, we have "Creatinine $\Perp$ Urine Flow $\mid$ Digoxin" and "Digoxin $\Perp$ Urine Flow $\mid$ Creatinine identified by both non-parametric tests. Due to the rule of learning adjacency, direct links between Urine Flow and both clearances are excluded. However, the kernel test confirmed "Urine Flow $\not 1$ (Creatinine, Digoxin)" with a p-value of 0.007, i.e., urine flow is dependent of clearances, which is indeed plausible from the medical viewpoint. Such inconsistence violates the so-called intersection property [20] of a BN structure, which states $\left(Y_{1} \Perp X \mid Y_{2}\right) \wedge\left(Y_{2} \Perp X \mid Y_{1}\right) \Rightarrow X \Perp\left(Y_{1}, Y_{2}\right)$. In fact, the intersection property does not hold in general. A trivial example for 
such violation is that $Y_{1}$ and $Y_{2}$ are related deterministically with each other (see 2122] for more theoretical discussions about the problem in learning BN with deterministic nodes), i.e., $Y_{1}$ and $Y_{2}$ contain entire information about each other. The uncertainty of $Y_{1}$ (or $Y_{2}$ ) vanishes due to the knowledge of $Y_{2}$ (or $Y_{1}$ ), then testing conditional dependences between $X$ and $Y_{1}$ given $Y_{2}$ and between $X$ and $Y_{2}$ given $Y_{1}$ cannot provide any evaluable information about the dependence between $X$ and $\left(Y_{1}, Y_{2}\right)$. Martín 23 showed that this property only holds, when $Y_{1}$ and $Y_{2}$ are measurably separated. The so-called "measurable separability" concept is introduced by Florens et al. 24] and provides a sufficient assumption to make the intersection property valid [23. Since such violation essentially reveals some symmetry in CI constraints between the measurably inseparable $Y_{1}$ and $Y_{2}$ w.r.t. $X$, we propose to consider $\left(Y_{1}, Y_{2}\right)$ as one factor in the resulting structure, which makes such conflicting information of CI constraints irrelevant. By means of kernel independence tests, structures containing vectorial variables can be straightforward handled. The following examples will show that such construction of nodes gains advantage in the structure learning, in particular, in relation to the potential causal interpretation of the structure.

Montana Outlook Poll. The data contain the outcome in the Montana Economic Outlook Poll in May 1992. This benchmark dataset is listed at http:// lib.stat.cmu.edu/DASL/Stories/montana.html. After removing records with missing values, the dataset contains 163 entries. The Montana poll asked a random sample of Montana residents whether the respondent feels his/her personal financial status is worse, the same, or better than a year ago, and whether they view the state economic outlook as better over the next year. Respondents are classified by sex, age, income, political orientation, and area of residence in the state. In the triple $\{$ Financial,Political,Outlook $\}$, we observe (Outl. $\Perp$ Fin. $\mid$ Pol.) and (Outl. $\Perp$ Pol. |Fin.). According to the rule of learning adjacency, the first two constraints excluded a direct link between Outl. and Fin., and a direct link between Outl. and Pol. (Fig. 4 left), which seems to be implausible, due to the constraint (Outl. $\not 1$ (Fin., Pol.)) identified by kernel test. If we merge Fin. and Pol. together to a new node, we will obtain indeed a structure (Fig. 4, right) which can be plausibly interpreted causally.

US Crime Data. The US crime data 25] are crime-related and demographic statistics for 47 US states in 1960. This dataset is listed as benchmark for causal structure learning on the homepage of TETRAD project. The dataset consists of
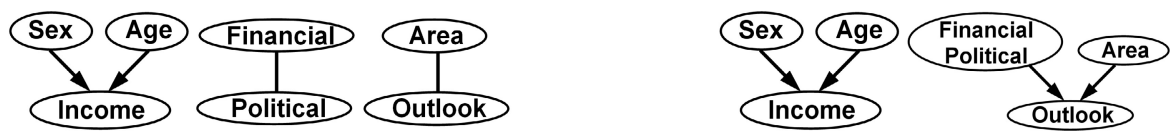

Fig. 4. Learning BN structure on Montana data. The left plot represents the structure learned with inconsistent CI constraints. The right plot illustrates a structure without representing the violation of the intersection property of a BN structure. 
14 variable: CRIME rate; the number of Young MALE; States: binary indicator variable for Southern states; EduCATION; Ex59, Ex60: 1959, 1960 per capita expenditure on police by state and local government; YOUNG LABOR force participation rate; the number of MALE; Population; the number of Non-WHites; U1: unemployment rate of urban males of age 14-24; U2: unemployment rate of urban males of age 35-39; AssETs: value of transferable goods and assets or family income; POVERTY: the number of families earning below 1/2 the median income. It is remarkable that the output of $\mathrm{PC}$ is not a $\mathrm{BN}$ contains 4 bi-directed edges (Fig. 5, left), which are traced back to the inconsistence of CI constraints. If we wish to find out the causal relationships between crime rate and other factors, the result of PC is unsatisfactory, although they provide some plausible connections between the expenditure on police and crime rate, some relationships among demographic statistics. Regarding the meaning of variables, it is obvious that some variables must be strongly related. In order to better understand the phenomenon of crime rate, we propose to reconstruct a demographic and geographic factor, called DEMo-GEOGRAPHIC (comprising Population, Non-White, Male, Young Male, and States), a factor called ExpendiTURE (containing Ex59 and Ex60), a factor called EMPLOYMENT (containing Young LABOR, U1, and U2), and a factor WeAlth (containing Assets and Poverty). The variable Crime remains unchanged. We conducted the so-called KCL algorithm [10] to learn the causal ordering of variables on the complete connected graph. After that, we used the kernel independence tests to remove the unnecessary edges. The output is shown in the right plot of Fig. 5. The variable CRIME is reasonably detected as the effect of other factors. Interestingly, CRIME is conditional independent of EMPLOYMENT given ExPEnditurE. This example demonstrates the main advantage of kernel tests, i.e., the possibility to analyze the relationship between vectorial factors on continuous, categorical, vectorial or mixed domains. Nonetheless, it is actually difficult to judge the performance of a structure learning algorithm by experiments with real-world data, where the ground truth is not completely known, although the outputs of previous examples seem to be plausible in relation to intuitive causal interpretation. For this reason, we conduct experiments with the kernel tests in comparison to other
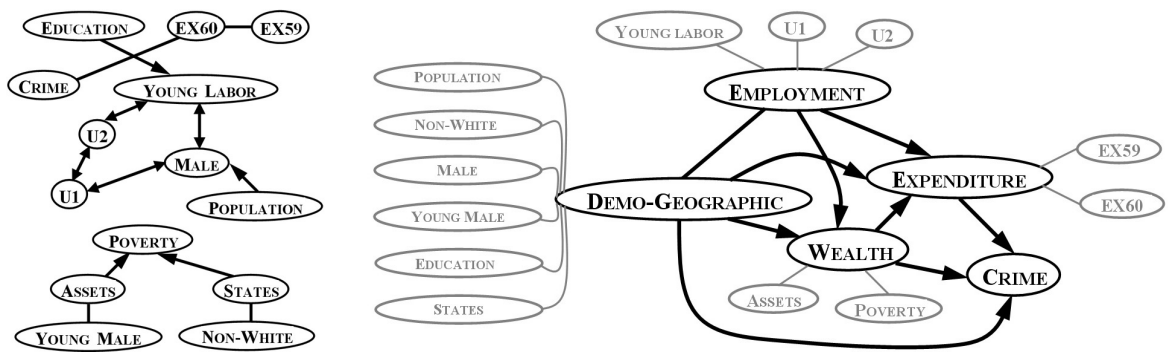

Fig. 5. Outputs learned by PC algorithm (left), KCL and kernel independence tests (right) from US crime data 
methods on purely categorical or continuous domains to get more evidence of performance improvements.

\subsection{Comparison of Performance on Categorical Domains}

First, we use toy data on categorical domains. The data are sampled from logically linked models, namely noisy OR gates. Such Boolean functions are simplified models for relations in many real-world situations. In general, an n-bit $X_{1}, \ldots, X_{n} \in\{0,1\}$ noisy OR gate can be characterized by the conditional probabilities $P\left(X_{n+1}=1 \mid x_{1}, \ldots, x_{n}\right)=\left(1-r_{2}\right)\left(1-r_{1}^{x_{1}+\ldots+x_{n}}\right)+r_{2}$ with parameters $r_{1}, r_{2} \in[0,1] . r_{1}$ can be interpreted as the probability of suppressing the input 1 ; $r_{2}$ can be interpreted as the probability for a spontaneous inversion of the output. If $r_{1}$ and $r_{2}$ vanish, the OR gate is deterministic. For the sake of notational simplicity, we chose $r_{1}=r_{2}=: r$ in this paper, i.e.,

$$
P\left(X_{n+1}=1 \mid x_{1}, \ldots, x_{n}\right)=(1-r)\left(1-r^{x_{1}+\ldots+x_{n}}\right)+r .
$$

Shorthand $\mathrm{OR}_{r}\left\{X_{1}, \ldots, X_{n}\right\}$ is used to depict a noisy OR gate with noise level $r \in[0,1]$. Data are sampled from a 2-bit noisy OR (Fig. 1 right) with $P(X=1)=$ 0.6 and $P(Y=1)=0.5$ as inputs and $Z=\mathrm{OR}_{r}\{X, Y\}$ as output. The underlying model implies $X \Perp Y$ and $X \not \Perp Y \mid Z$. We sampled 1000 datasets with different noise levels $r=0.0,0.1,0.2,0.3$ and different sample sizes 20,50, 100, 150, 200 . Fig. 6] shows the real noise statistics in term of percentage of erroneous outputs in 1000 data points. We perform the permutation test via kernel dependence measure (KD) and two popular independence tests on categorical domains, i.e., likelihood ratio $\chi^{2}$ test, permutation test via MI. Since all these three CI tests are non-parametric, their performance are expected to be similar, in the sense
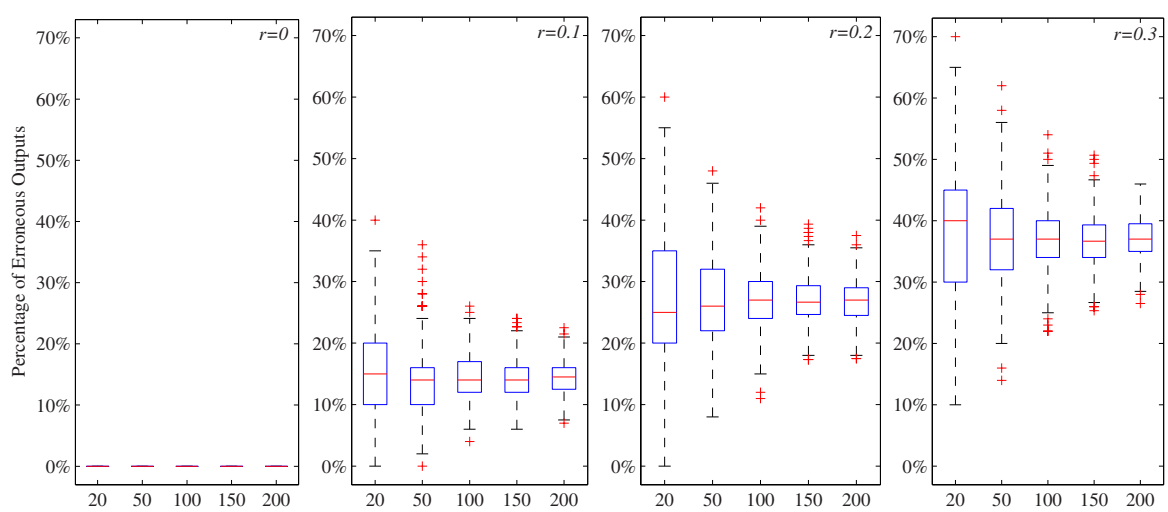

Fig. 6. Noise statistics in the term of percentage of erroneous outputs in 1000 data points sampled by 2-bit noisy OR gates. The plots illustrate 4 different noise levels $r=0,0.1,0.2,0.3$ as in Eq. (1). Each box has lines at the lower quartile, median, and upper quartile values of the percentage of erroneous outputs. The whiskers are lines extending from each end of the box to show the extent of the rest of the percentage. Outliers are the percentage beyond the ends of the whiskers. 
Table 3. Numerical comparison of three different independence tests on categorical domain, i.e., likelihood ratio $\chi^{2}$ test, permutation test via mutual information (MI), and permutation test via kernel dependence (KD) measure. The generating models are noisy OR gates with 4 different noise levels $r=0.0,0.1,0.2,0.3$ as shown in Eq. (1). The experiments are conducted with 1000 replications. The entries show how often (in percentage) the constraint $X \Perp Y$ or $X \not \Perp Y \mid Z$ is identified.

\begin{tabular}{|c|c|c|c|c|c|c|c|c|c|c|c|c|c|c|c|}
\hline & \multicolumn{15}{|c|}{ Accepting $X \Perp Y$} \\
\hline Sample Size & \multicolumn{3}{|c|}{20} & \multicolumn{3}{|c|}{50} & \multicolumn{3}{|c|}{100} & \multicolumn{3}{|c|}{150} & \multicolumn{3}{|c|}{200} \\
\hline Noise Level & $\chi^{2}$ & MI & $\mathrm{KD}$ & $\chi^{2}$ & MI & $\mathrm{KD}$ & $\chi^{2}$ & MI & KD & $\chi^{2}$ & $\mathrm{MI}$ & $\mathrm{KD}$ & $\chi^{2}$ & $\mathrm{MI}$ & $\mathrm{KD}$ \\
\hline$r=0.0$ & 94.0 & 97.4 & 88.0 & 94.7 & 96.3 & 90.8 & 95.6 & 95.9 & 92.5 & 94.3 & 93.7 & 92.4 & 94.1 & 94.3 & 91.8 \\
\hline$r=0.1$ & 93.1 & 96.4 & 86.5 & 94.6 & 96.0 & 90.7 & 94.2 & 94.0 & 91.1 & 95.8 & 96.3 & 94.4 & 94.2 & 94.8 & 92.3 \\
\hline$r=0.2$ & 93.6 & 96.9 & 86.9 & 94.9 & 96.1 & 91.3 & 96.3 & 96.1 & 93.1 & 95.7 & 95.7 & 93.5 & 93.6 & 94.0 & 91.4 \\
\hline$r=0.3$ & 94.5 & 97.1 & 87.3 & 95.9 & 97.0 & 93.0 & 93.5 & 93.6 & 90.7 & 93.6 & 94.1 & 91.6 & 94.4 & 94.8 & 93.2 \\
\hline Noise Level & \multicolumn{15}{|c|}{ Rejecting $X \Perp Y \mid Z$} \\
\hline$r=0.0$ & 24.8 & 54.8 & 23.5 & 94.5 & 97.7 & 91.9 & 100 & 100 & 98.0 & 100 & 100 & 100 & 100 & 100 & 100 \\
\hline$r=0.1$ & 23.5 & 33.7 & 16.9 & 57.6 & 57.0 & 54.8 & 85.9 & 84.7 & 89.2 & 97.6 & 97.3 & 98.0 & 99.3 & 99.2 & 99.7 \\
\hline$r=0.2$ & 14.9 & 18.9 & 8.9 & 25.1 & 22.7 & 22.5 & 39.8 & 40.7 & 40.5 & 56.2 & 57.2 & 60.0 & 71.6 & 72.5 & 74.8 \\
\hline$r=0.3$ & 9.9 & 10.9 & 7.0 & 10.4 & 10.3 & 9.6 & 16.3 & 16.5 & 16.3 & \begin{tabular}{|l|}
19.1 \\
\end{tabular} & 21.5 & 19.2 & 23.1 & 23.9 & 23.9 \\
\hline
\end{tabular}

that the levels of type I and II errors are almost the same. The larger the sample size and the less noisy the model, the better the performance (see Tab. 3 for results). The kernel test is slightly worse than the other two in the case of 20 data points. The actual benefit of kernel tests does not lie in the tests on categorical domains, but in tests on continuous or hybrid domains. Nonetheless, the kernel independence test provides a good alternative to the popular tests on categorical domains.

\subsection{Comparison of Performance on Continuous Domains}

Toy data are generated by functional models on continuous domains. Fisher's Z test, Margaritis' Bayesian method and permutation test via kernel dependence measures are evaluated. Although the estimation of MI on continuous domains is an unsolved problem in its generality, there are some attempt to do that. We performed permutation test via MI using the estimation method proposed by Kraskov et al. [8]. First, we sampled the so-called Meander data. The generating model of Meander data is proposed by Margaritis 26. It resembles a spiral (Fig. 7). This dataset is challenging because the joint distribution of $X$ and $Y$ given $Z$ changes dramatically with the given value of $Z$. According to the functional relation, $X$ and $Y$ are conditionally independent given $Z$, however, unconditionally dependent, in fact strongly correlated (rightmost plot of Fig. 7). We generated 1000 datasets and ran tests. Tab. 4 shows the results for samples sizes ranging from 20 to 200. The dependence between $X$ and $Y$ can already be captured by the linear relation. All methods achieved already very good performance at testing $X \not \Perp Y$ from merely 20 data points. Testing 


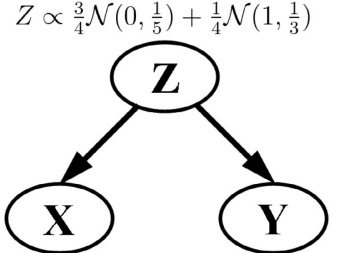

$X \propto \frac{Z}{10}+\frac{1}{2} \sin (2 \pi Z)+0.1 \mathcal{N}(0,1)$

$Y \propto \frac{Z}{5}+\sin (2 \pi Z+0.35)+0.1 \mathcal{N}(0,1)$
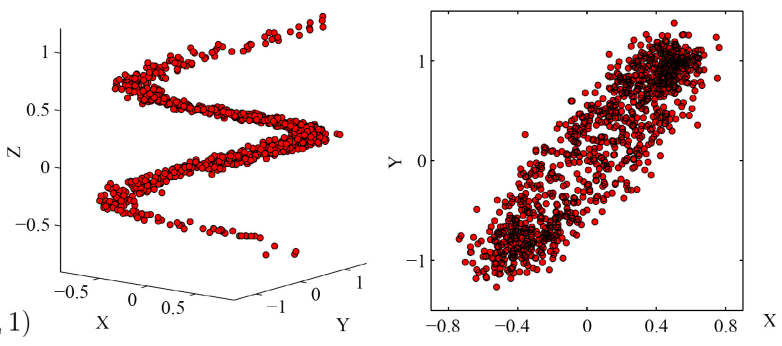

Fig. 7. Generating model of meander dataset (leftmost), which implies $X \not \Perp L Y$ and $X \Perp Y \mid Z$. 3-dimensional plot of the Meander dataset (middle) and projection of data along $Z$ axis (rightmost)

Table 4. Comparison of independence tests on continuous domains, i.e., Fisher's Z test, Margaritis' Bayesian method, and permutation test via mutual information and kernel dependence measures. The underlying model Meander is given by Fig. 7 The experiments are conducted with 1000 replications. The entries show how often (in percentage) the constraint $X \not \Perp Y$ and $X \Perp Y \mid Z$ are identified.

\begin{tabular}{|c||c|c|c|c|c||c|c|c|c|c|}
\hline \multicolumn{1}{|c||}{} & \multicolumn{3}{c||}{ Rejecting $X \Perp Y$} & \multicolumn{4}{c|}{ Accepting $X \Perp Y \mid Z$} \\
\hline Sample Size & 20 & 50 & 100 & 150 & 200 & 20 & 50 & 100 & 150 & 200 \\
\hline \hline Fisher's Z & 100 & 100 & 100 & 100 & 100 & 0 & 0 & 0 & 0 & 0 \\
\hline Margaritis' Bayesian & 94.3 & 100 & 100 & 100 & 100 & 4.8 & 15.1 & 21.2 & 23.2 & 33.2 \\
\hline Mutual Information & 99.6 & 100 & 100 & 100 & 100 & 8.6 & 19.4 & 33.8 & 38.9 & 43.7 \\
\hline Kernel Dependence & 99.9 & 100 & 100 & 100 & 100 & 35.1 & 49.7 & 67.0 & 75.3 & 79.9 \\
\hline
\end{tabular}

\begin{tabular}{|l||c|c|c|c|}
\hline$M_{k}:=\left(f_{i}, f_{j}\right)$ & $f_{2}:=2 \sin (x)$ & $f_{3}:=\ln (|x|)$ & $f_{4}:=\frac{1}{\frac{x}{5}+1}$ & $f_{5}:=\exp (x)$ \\
\hline \hline$f_{1}:=x$ & $M_{1}$ & $M_{2}$ & $M_{3}$ & $M_{4}$ \\
\hline$f_{2}:=2 \sin (x)$ & - & $M_{5}$ & $M_{6}$ & $M_{7}$ \\
\hline$f_{3}:=\ln (|x|)$ & - & - & $M_{8}$ & $M_{9}$ \\
\hline$f_{4}:=\frac{1}{\frac{x}{5}+1}$ & - & - & - & $M_{10}$ \\
\hline
\end{tabular}

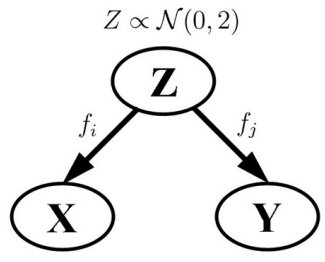

$X \propto f_{i}(Z)+\mathcal{N}(0,1) \quad Y \propto f_{j}(Z)+\mathcal{N}(0,1)$
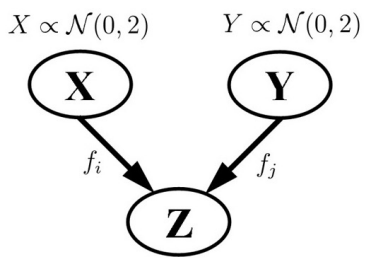

$Z \propto f_{i}(X)+f_{j}(Y)+\mathcal{N}(0,1)$

Fig. 8. Functional models with a $\wedge$-structure (left plot) and a $\vee$-structure (right plot). The pairs of functions $M_{k}=\left(f_{i}, f_{j}\right)$ with $i, j=1, \ldots, 5$ and $k=1, \ldots, 10$ for both structures are defined in the table. 

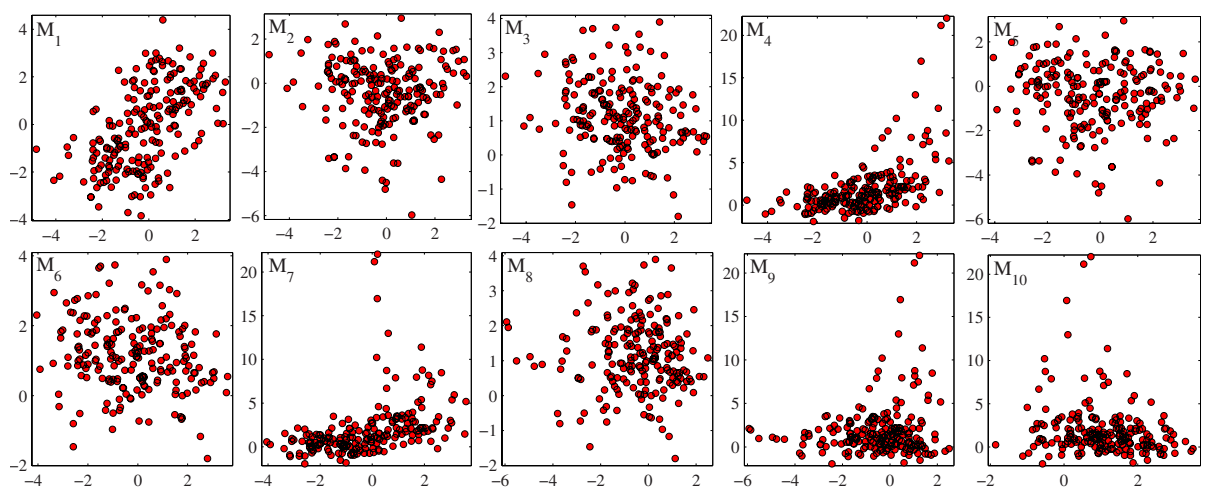

Fig. 9. 2-dimensional plots of data sampled from a $\wedge$-structure (Fig. 8 first plot), where $X$ and $Y$ have a functional relation $M_{k}=\left(f_{i}, f_{j}\right)$ (see table in Fig. 8) with $Z$, respectively. The illustrated sample contains 200 data points.

Table 5. Comparison of various independence tests on continuous domains sampled by a $\wedge$-structure (Fig. 8 , first plot). The parameter $M_{k}=\left(f_{i}, f_{j}\right)$ of models is defined in the table in Fig. 8 . The entries show how often (in percentage) $X \not \Perp Y$ and $X \Perp Y \mid Z$ are identified after 1000 replications of simulations.

\begin{tabular}{|c||c|c|c|c|c|c|c|c|c|c|}
\hline \multicolumn{1}{|c||}{} & \multicolumn{10}{|c|}{ Rejecting $X \Perp Y$} \\
\hline$M_{k}=\left(f_{i}, f_{j}\right)$ & $M_{1}$ & $M_{2}$ & $M_{3}$ & $M_{4}$ & $M_{5}$ & $M_{6}$ & $M_{7}$ & $M_{8}$ & $M_{9}$ & $M_{10}$ \\
\hline \hline Fisher's Z & 100 & 4.2 & 93.6 & 100 & 1.8 & 57.6 & 71.5 & 17.5 & 41.0 & 58.6 \\
\hline Margaritis' Bayesian & 100 & 2.0 & 42.5 & 100 & 2.0 & 27.6 & 100 & 1.7 & 4.0 & 18.6 \\
\hline Mutual Information & 91.0 & 43.2 & 31.9 & 100 & 33.3 & 60.2 & 100 & 5.2 & 65.5 & 9.2 \\
\hline Kernel Dependence & 100 & 95.6 & 63.9 & 100 & 63.9 & 56.1 & 100 & 11.5 & 97.8 & 68.7 \\
\hline \hline \multicolumn{1}{|c||}{} & \multicolumn{10}{|c|}{ Accepting $X \Perp Y \mid Z$} \\
\hline Fisher's Z & 94.0 & 95.6 & 94.1 & 95.6 & 95.5 & 72.2 & 10.6 & 81.5 & 1.2 & 64.3 \\
\hline Margaritis' Bayesian & 97.0 & 97.6 & 97.9 & 98.7 & 97.0 & 97.9 & 98.9 & 98.3 & 98.7 & 98.8 \\
\hline Mutual Information & 80.1 & 92.5 & 93.6 & 88.4 & 85.7 & 92.9 & 88.2 & 97.5 & 93.6 & 89.4 \\
\hline Kernel Dependence & 93.8 & 93.8 & 92.5 & 93.4 & 93.3 & 93.5 & 93.4 & 94.5 & 94.2 & 92.9 \\
\hline
\end{tabular}

conditional independence $X \Perp Y \mid Z$ is more challenging. Here, the kernel test clearly outperforms other three methods. Fisher's Z test fails completely due to the incorrect multivariate Gaussian assumption. The kernel independence test made significantly less errors than Margaritis' Bayesian method and test via MI. In order to gain more evidence of performance in learning BN structure, we sampled datasets of 200 data points by different functional models with the $\wedge$ and $\vee$-structures (Fig. 8). We define the functional relations $f_{1, \ldots, 5}$ in the same way as proposed in [26] and use all pairs of them $M_{k}=\left(f_{i}, f_{j}\right)$, i.e., 10 different combinations $M_{1}, \ldots, M_{10}$ as shown in the table in Fig. 8, added by a Gaussian noise as underlying ground-truth for the sampling. One sample of 200 data points for the $\wedge$-structure (left plot in Fig. 8) with $M_{1}, \ldots, M_{10}$ is visualized in Fig. 9 and Fig. 10. The performance of various independence tests after 1000 

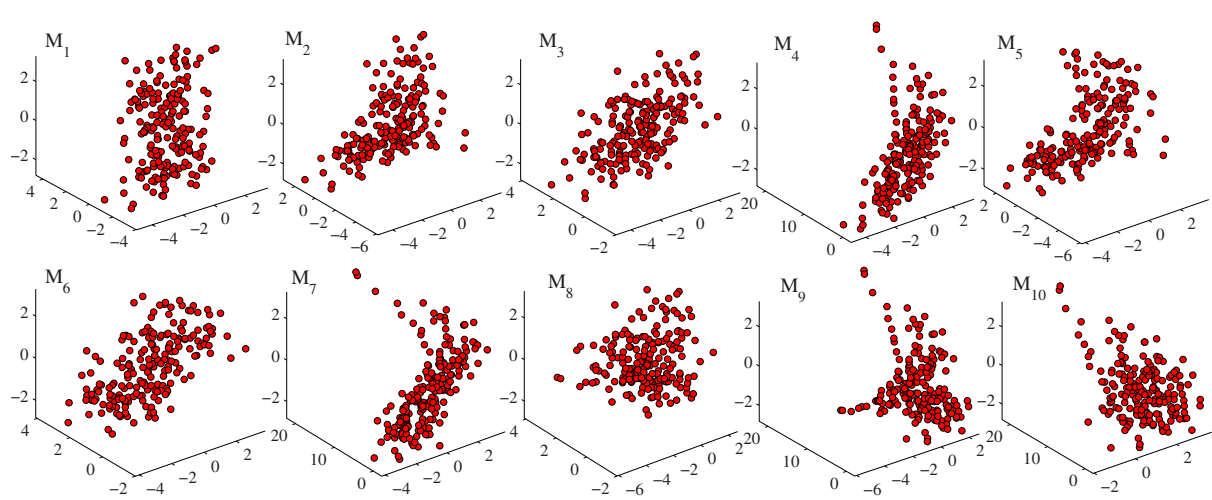

Fig. 10. 2-dimensional plots of data sampled from a $\wedge$-structure (Fig. 8 first plot), where $X$ and $Y$ have a functional relation $M_{k}=\left(f_{i}, f_{j}\right)$ (see table in Fig. 8) with $Z$, respectively. The illustrated sample contains 200 data points.

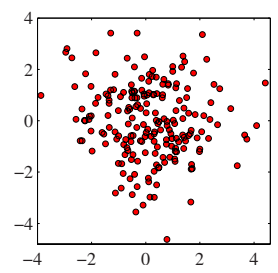

Fig. 11. 2-dimensional plots of data sampled from a $\vee$-structure (Fig. 8 second plot), where $Z$ has a functional relation $M_{k}=\left(f_{i}, f_{j}\right)$ (see table in Fig. 8) with $X$ and $Y$. The illustrated sample contains 200 data points.
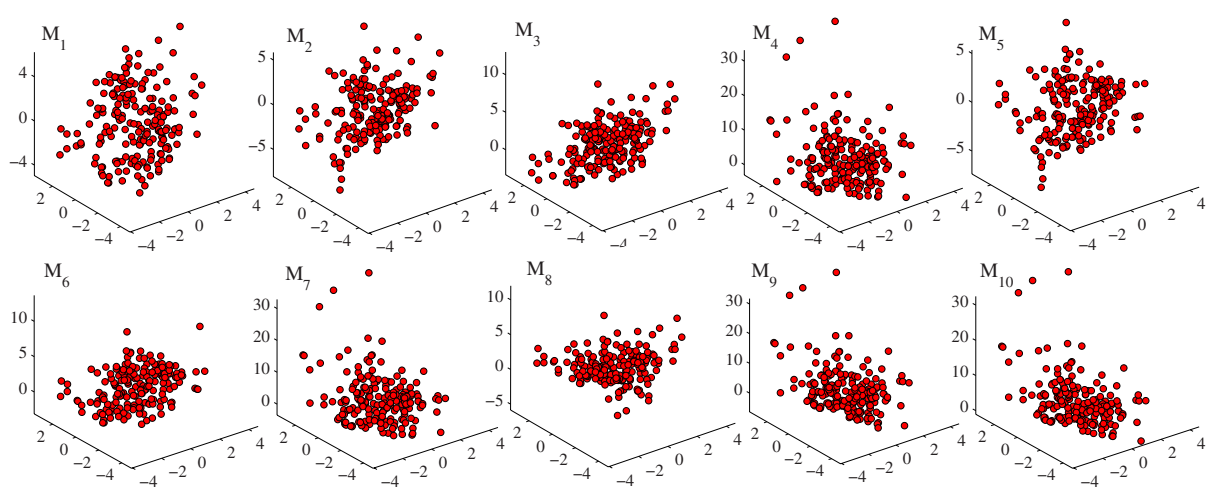

Fig. 12. 3-dimensional plots of data from a $\vee$-structure (Fig. 8 second plot), where $Z$ has a functional relation $M_{k}=\left(f_{i}, f_{j}\right)$ (see table in Fig. 8) with $X$ and $Y$. The illustrated sample contains 200 data points. 
Table 6. Comparison of various independence tests on continuous domains sampled by a $\wedge$-structure (Fig. 8 , first plot). The parameter $M_{k}=\left(f_{i}, f_{j}\right)$ of models is defined in the table in Fig. 8. The entries show how often (in percentage) $X \Perp Y$ and $X \not \Perp Y \mid Z$ are identified after 1000 replications of simulations.

\begin{tabular}{|c||c||c|c|c|c|c|c|c|c|c|c|}
\hline \multicolumn{1}{|c||}{} & Accepting $X \Perp Y$ & \multicolumn{10}{|c|}{ Rejecting $X \Perp Y \mid Z$} \\
\hline$M_{k}=\left(f_{i}, f_{j}\right)$ & $M_{1, \ldots, 10}$ & $M_{1}$ & $M_{2}$ & $M_{3}$ & $M_{4}$ & $M_{5}$ & $M_{6}$ & $M_{7}$ & $M_{8}$ & $M_{9}$ & $M_{10}$ \\
\hline \hline Fisher's Z & 94.6 & 100 & 4.1 & 92.1 & 77.1 & 4.7 & 58.8 & 61.2 & 5.1 & 3.9 & 20.8 \\
\hline Margaritis' Bayesian & 98.1 & 91.4 & 3.9 & 10.9 & 84.8 & 3.1 & 9.1 & 75.0 & 2.1 & 3.7 & 6.7 \\
\hline Mutual Information & 92.3 & 92.3 & 66.8 & 17.5 & 76.9 & 65.6 & 16.9 & 76.3 & 1.2 & 21.9 & 8.0 \\
\hline Kernel dependence & 94.1 & 100 & 92.0 & 60.7 & 100 & 96.0 & 51.7 & 100 & 18.7 & 93.6 & 46.9 \\
\hline
\end{tabular}

replications on these datasets is summarized in Tab. 5. One sample of 200 data points for the $\mathrm{V}$-structure (right plot in Fig. 8) with $M_{1}, \ldots, M_{10}$ is visualized in Fig. 11 and Fig. 12, The performance of various independence tests of 1000 replications on these datasets is summarized in Tab. 6.

One can see that all methods make relatively few errors at discovering independence, i.e., $X \Perp Y$ in the $\vee$-structure (left half of Tab. 6) and $X \Perp Y \mid Z$ in the $\wedge$-structure (under half of Tab. 5). Fisher's Z test performed very bad in the case of testing conditional independence on data sampled by models $M_{7}$ and $M_{9}$ (first row of the under half of Tab. 5). In summary, the kernel tests outperformed the other non-parametric approaches, i.e., Margaritis' Bayesian method and test via mutual information, in cases of testing dependence, i.e., $X \not \Perp Y$ in the $\wedge$-structure (upper half of Tab. 5) and $X \not \Perp Y Y \mid Z$ in the $\vee$-structure (see the right half of Tab. 6). In addition, the results indicate that the fluctuation of the kernel tests within different models is significantly smaller than that of the correlation analysis based on the linear model. For this reason, we can reasonably expect more accuracy and reliability in testing CI constraints, and consequently better performance in identification of $\wedge$ - and $\vee$-structures, thus structure learning in general by using kernel tests in existing independence-based algorithms.

\section{Conclusion}

A new method for structure learning of Bayesian networks with arbitrary distributions on arbitrary domains is demonstrated. This was made possible by the use of the probabilistic non-parametric conditional independence tests presented in the paper. Our evaluation on both real and artificial data shows that our method performs well against existing alternatives. Using kernel independence tests for learning Bayesian network structure is expected to make less errors than other state-of-the-art methods used in the independence-based algorithms. 


\section{References}

1. Heckerman, D., Meek, C., Cooper, G.: A Bayesian approach to causal discovery. In: Glymour, C., Cooper, G. (eds.) Computation, Causation, and Discovery, pp. 141-165. MIT Press, Cambridge (1999)

2. Cooper, G.: The computational complexity of probabilistic inference using Bayesian belief networks. Journal of Artificial Intelligence 42(3-4), 393-405 (1990)

3. Chickering, D., Heckerman, D., Meek, C.: Large-sample learning of Bayesian networks is NP-hard. Journal of Machine Learning Research 5, 1287-1330 (2004)

4. Spirtes, P., Glymour, C., Scheines, R.: Causation, prediction, and search. Lecture notes in statistics. Springer, New York (1993)

5. Pearl, J.: Causality: Models, reasoning, and inference. Cambridge University Press, Cambridge (2000)

6. Margaritis, D.: A Bayesian multiresolution independence test for continuous variables. In: Proceedings of the 17th conference on uncertainty in artificial intelligence, Pittsburgh, PA, pp. 346-353 (2001)

7. Margaritis, D.: Distribution-free learning of Bayesian network structure in continuous domains. In: Proceedings of the 20th National Conference on Artificial Intelligence, Seattle, WA, pp. 825-830 (2005)

8. Kraskov, A., Stögbauer, H., Grassberger, P.: Estimating mutual information. Physical Review E 69(6), 66138 (2000)

9. Schölkopf, B., Smola, A.: Learning with kernels. MIT Press, Cambridge (2002)

10. Sun, X., Janzing, D., Schölkopf, B., Fukumizu, K.: A kernel-based causal learning algorithm. In: Ghahramani, Z. (ed.) Proceedings of the 24th International Conference on Machine Learning, Corvallis, OR, pp. 855-862 (2007)

11. Fukumizu, K., Gretton, A., Sun, X., Schölkopf, B.: Kernel measures of conditional dependence. In: Platt, J., Koller, D., Singer, Y., Roweis, S. (eds.) Proceedings of the 21th Neural Information Processing Systems Conference, pp. 489-496. MIT Press, Cambridge (2007)

12. Gretton, A., Bousquet, O., Smola, A., Schölkopf, B.: Measuring statistical dependence with Hilbert-Schmidt norms. In: Jain, S., Simon, H.U., Tomita, E. (eds.) ALT 2005. LNCS (LNAI), vol. 3734, pp. 63-77. Springer, Heidelberg (2005)

13. Good, P.: Permutation tests: A practical guide to resampling methods for testing hypothesis. Birkhäuer, Boston (1994)

14. Fine, S., Scheinberg, K.: Efficient SVM training using low-rank kernel representations. Journal of Machine Learning Research 2, 243-264 (2001)

15. Gretton, A., Fukumizu, K., Teo, C., Song, L., et al.: A kernel statistical test of independence. In: Platt, J., Koller, D., Singer, Y., Roweis, S. (eds.) Proceedings of the 21th Neural Information Processing Systems Conference, pp. 585-592. MIT Press, Cambridge (2007)

16. Halkin, H., Sheiner, L., Peck, C., Melmon, K.: Determinants of the renal clearance of digoxin. Clinical Pharmacology and Therapeutics 17(4), 385-394 (1975)

17. Altman, D.G.: Practical statistics for medical research. Chapman and Hall, London (1991)

18. Edwards, D.: Introduction to graphical modelling. Springer, New York (2000)

19. Jelliffe, R., Blankenhorn, D.: Improved method of digitalis therapy in patients with reduced renal function. Circulation 35, 11-150 (1967)

20. Pearl, J.: Probabilistic reasoning in intelligent systems: Networks of plausible inference. Morgan Kaufmann, San Mateo (1988) 
21. Shachter, R.: Probabilistic inference and influence diagrams. Operations Research 36(4), 589-604 (1988)

22. Geiger, D.: Graphoids: A qualitative framework for probabilistic inference. PhD thesis, Cognitive Systems Laboratory, Department of Computer Science, University of California, Los Angeles, CA (1990)

23. Martín, E.: Ignorable common information, null sets and Basu's first theorem. The Indian Journal of Statistics 67(4), 674-698 (2005)

24. Florens, J., Mouchart, M., Rolin, J.: Elements of Bayesian Statistics. Marcel Dekker, New York (1990)

25. Vandaele, W.: Participation in illegitimate activites: Erlich revisited. In: Blumstein, A., Cohen, J., Nagin, D. (eds.) Deterrence and incapacitation, pp. 270-335. National Academy of Sciences, Washington (1978)

26. Margaritis, D.: Distribution-free learning of graphical model structure in continuous domains. Technical Report TR-ISU-CS-04-06, Computer Science, Iowa State University (2004) 